\title{
What are the non-food impacts of GM crop cultivation on farmers' health?
}

\author{
Monica Racovita ${ }^{1,3^{*}}$, Dennis Ndolo Obonyo ${ }^{2}$, Wendy Craig ${ }^{1}$ and Decio Ripandelli ${ }^{1}$
}

\begin{abstract}
Background: Although approved for commercialisation in a number of countries since the 1990s, the potential environmental, human/animal health, and socio-economic impacts of genetically modified (GM) crops are still widely debated. One category of human health impacts (designated in this review as non-food health impacts) focuses on indirect effects of GM crop cultivation; amongst which the most prominent are health benefits via: (1) reduced use of pesticides, and (2) an increase in income. Both of these pathways have raised a lot of interest in the developing world, especially in areas experiencing high rates of pesticide poisonings and low agricultural incomes. However, evidence to support such benefits has been relatively scarce in comparison to that of GM food health impacts. Non-food health impacts of GM crop cultivation on farmers deserve more attention, not just because of an apparent knowledge gap, but also because of, potential economic and environmental implications, involving for example $\mathrm{CO}_{2}$ emissions, underground water contamination and improved sanitation.
\end{abstract}

Methods/Design: The primary research question was: What are the non-food impacts of GM crop cultivation on farmers' health? To address this primary question, the study focused on two related secondary questions: (1) Does the cultivation of GM crops result in a lower number of pesticide-related poisonings as compared to the cultivation of their non-GM counterparts?, and; (2) Does the cultivation of GM crops allow for higher financial resources to be used by farmers to improve the health status of themselves and their family, as compared to the cultivation of the non-GM counterpart? The extent to which information relevant to the two secondary questions was freely-available was also evaluated. The search and assessment methodologies were adapted following experience gained during a scoping exercise, and followed the published protocol.

Results: The 20 databases and 10 reviews searched returned 4,870 hits, with 19 identified as relevant for data extraction. It was apparent that the 19 articles were derived from only 9 original studies, of which 7 were relevant to the first research question, whilst the remaining 2 were relevant to the second question. The studies showed both an overall decrease in the amount of pesticides applied and an increase in household income from GM crop cultivation as compared to the cultivation of the non-GM counterpart.

Conclusion: In the absence of additional confounding variables or statistical analyses to support these findings, any correlation from these studies should be considered circumstantial at best. Even though the cultivation of GM crops appears to increase household income, evidence to demonstrate that farmers invested this extra income in improving their health remained inconclusive. Further research is therefore needed to clarify the possible correlation between GM crop cultivation and (1) pesticide poisonings, and (2) overall health improvements. Future impact evaluations should include: both written records and surveys; statistical correlations between independent and dependent variables; testing the characteristics of the samples for statistical significance to indicate their representativeness of

\footnotetext{
*Correspondence: mracovita@gmail.com

${ }^{3}$ Present Address: STS-Institute of Science, Technology and Society

Studies Alpen-Adria-Universität Klagenfurt, Vienna, Graz, Schlögelgasse 2,

8010 Graz, Austria

Full list of author information is available at the end of the article
} 
a particular population, and; increasing the importance of confounding variables in research design (by identifying specific variables and selecting sample and control groups accordingly).

Keywords: GM crops, Farmers, Pesticides, Poisonings, Incomes, Health expenditures

\section{Background}

The first GM crop approved for commercialisation was a delayed-ripening tomato in 1994 in the USA. By 2014 there were 28 countries growing GM crops on a cumulative area of 181 million hectares, making GM crops the technology with the fastest rate of uptake in modern agriculture [1]. However, many contradictory reports have been published concerning the impacts of GM crop cultivation. Whilst some have pointed towards an array of socio-economic and environmental advantages, others have indicated that GM crop cultivation comes with adverse consequences. For example, in addition to an increase in farm income [2], the cultivation of GM crops has been reported to reduce the environmental impact of agriculture through: lowering the amount of pesticides applied [3]; decreasing the amount of fossil fuel consumed and $\mathrm{CO}_{2}$ emitted due to a reduction or avoidance of ploughing [4], and; facilitating conservation tillage, resulting in better maintenance of agricultural soils [5, 6]. Conversely, other reports have indicated that the cultivation of GM crops resulted in: an increase in the use of pesticides [7]; failures to provide significantly increased yields [8], and; an increase in allergenic and toxic effects [9].

Within the vast array of impacts ascribed to GM crop cultivation, those pertaining to human health may not be as easily analysed. Effects on human health can be considered separately or included in economic or environmental evaluations [4]. Exposure pathways determine how health effects are considered, either (1) directly, due to the consumption of GM food [10], or direct contact with the plant during harvest, or (2) indirectly, depending on the influence of GM crop cultivation on on-farm pesticide use [11], or impacts on overall welfare related to changes in on-farm incomes. Where health effects have been included in economic evaluations, both pecuniary and non-pecuniary effects have been described [4]. In other instances, impacts on human health have been considered under broader environmental impacts, within indicators such as Environmental Impact Quotients (EIQs) [4].

The choice of research objectives for the present systematic review has been guided by the links between agricultural productivity and health. Such links include health impacts derived from changes in household income and from impacts on agricultural production chains generated by the introduction of new crops [12].
Any modification of household income can affect patterns of spending within the household, including the money available for buying more and/or better food, paying for medical expenses, and saving [12]. The introduction of a new crop can bring changes to: (1) consumption patterns of other agricultural commodities; (2) agricultural practices e.g. time assigned to on-farm work can interfere with other activities, or an intensification of agriculture practices with heavy use of pesticides and fertilisers which in turn can increase the number of accidents and toxicity from pesticides, and; (3) markets (availability of better/worse products than before) [12].

In addition, in addressing the human health impacts of GM crops, the focus is usually on effects resulting from the consumption of GMOs and their derived agricultural products. Non-food effects on human health are generally overlooked or ignored. In the few instances when they are addressed, the non-food impacts are not tackled in a separate category but rather variables pertaining to such impacts are included selectively in other categories of GMO impacts, such as those pertaining to the environment $\left(\mathrm{CO}_{2}\right.$ emissions, underground water contamination) or economics (improved sanitation). Further, due to the controversies surrounding GM crops, the objectivity of articles analysing the impacts of GM crop cultivation are often questioned. Amongst the latter category are reports that infer human health benefits from a reduction in pesticide use without performing any analyses to support the position [13]. For these reasons, a systematic review was chosen as the most appropriate methodology to guide the evaluation of the existing evidence of GM crop non-food effects on human health and/or to point to a gap(s) in existing knowledge.

This systematic review considered the impacts of GM crop cultivation arising from changes in on-farm pesticide usage and household income, and the resulting effects on health. As the primary beneficiaries of GM crop technology to date, farmers were the population selected for this study. "Farmers" were considered to include farm owners, farm labourers and agricultural contractors in developing and developed countries. As the focus of the systematic review concerned non-food impacts on GM crop cultivators rather than more general indirect impacts, this review did not investigate the possible contribution of GM crop cultivation to the lowering of greenhouse gas emissions, sometimes considered as an indirect health effect [4], nor did it explore other indirect 
effects such as the contamination of underground water or impacts on sanitation.

\section{Objectives}

The primary research question was: What are the nonfood impacts of GM crop cultivation on farmers' health? To specifically address the primary research question, the study focused on two related secondary questions: (1) Does the cultivation of GM crops result in a lower amount of pesticide usage compared to the cultivation of non-GM crops, hence leading to a reduction in pesticide-related poisonings?, and (2) Does the cultivation of GM crops allow for greater financial resources to be used by farmers to improve the health of themselves and their family, compared to farmers cultivating nonGM crops? Both secondary questions looked at final outcomes (number of pesticide-related poisonings and health-related expenditures) through a well-established pathway, via intermediary outcomes (amount of pesticide usage and income derived from GM crop cultivation). The main concepts addressed in this systematic review ("PICO"; P-Population, I-Intervention, C-Comparator, O-Outcome) [14-17] were formulated as shown in Table 1 of the published protocol [18].

The review also evaluated the extent to which information relevant to the two secondary questions was freelyavailable (understood to be data that could be accessed without expense, i.e. publicly-accessible materials from the Internet, databases routinely available to universities and public research institutions on a no-cost basis, as well as articles provided freely by the authors when contacted).

\section{Methods/Design}

\section{Searches}

The following combination of keywords and wildcard symbols (", \$), linked with Boolean operators 'AND' and 'OR' were used to search online databases: (farmer" OR agric* OR worker OR contractor) AND ([genetic"modif*] OR [genetic*engineer*] OR GE OR transgene* OR herbicide\$tolerant OR insect\$resistant OR biotech* OR BT OR GM OR stacked\$trait*) AND (crop* OR plant* OR flower") AND (health OR poisoning OR security OR safety OR welfare OR benefit) AND (pesticides OR insecticides OR herbicides OR fungicides OR bactericides OR income OR revenue OR gains OR wage OR profit OR earnings OR livelihood).

The composition of the search string was established following the experiences gained during a scoping exercise preceding the publishing of the protocol. At the time, a short combination of key words was employed to search in four databases. Afterwards the search string was improved with more synonyms (almost doubled the length of the search string), which increased the number of hits. The search string was established to reach a balance between comprehensiveness (reaching as many relevant hits as possible) and manageability (databases cannot handle searches with too many key words).

A special attention was given to the hits in Spanish or Portuguese. Since we employed databases that had abstracts in English for articles published in English, Spanish and Portuguese, we could use a search with English key words, yet retrieve articles in all three languages. The number of articles in English far exceeded the articles in Spanish or Portuguese. Combined with the fact that the language competencies of most of the members of the review team did not include Spanish or Portuguese, it was established that the search would focus only on articles in English.

The structure of the search string followed a PICO structure, albeit in a slightly modified form. The Intervention was represented by the GM crop cultivation. However, to ensure that the search terms referring to the Intervention were as inclusive as possible but yet captured only GM crops (and not GM insects or animals), "GM" and "crop" were separated into different categories. No string of key words was dedicated to the Comparator, as it was already included in the Intervention section: (crop* OR plant* OR flower*).

The Outcomes category was further separated into intermediary (pesticides OR insecticides OR herbicides OR fungicides OR bactericides OR income OR revenue OR gains OR wage OR profit OR earnings OR livelihood) and final outcomes (health OR poisoning OR effects OR welfare OR environment).

A list of databases (Table 1) was compiled after consulting the Cochrane Handbook for Systematic Reviews of Interventions [14], and further supplemented with additional databases suggested by the reviewers of our protocol. Some databases containing "grey literature" were also included i.e. literature produced in print and electronic formats by all levels of government, academics, business and industry but which are not controlled by commercial publishers. All selected databases were free for public use or could become so following online registration for a free trial. In addition, the cited literature of 10 reviews identified during the scoping exercise done at the protocol stage were also examined.

The databases were divided amongst three reviewers, with two reviewers searching each database. The databases were searched within 'title', 'topics' and 'full text' categories. As some databases did not accept the full search string, the reviewers were at liberty to adapt the search string to contain combinations of fewer words (for example, one term from each group within brackets united by an 'AND' operator). As this was 
Table 1 List of databases and collections searched

\begin{tabular}{|c|c|c|}
\hline & Name & Address \\
\hline \multirow[t]{9}{*}{ General } & BioMed Central & http://www.biomedcentral.com/browse/journals/ \\
\hline & Database of Promoting Health Effectiveness Reviews (DoPHER) & http://eppi.ioe.ac.uk/webdatabases/Intro.aspx?ID=2 \\
\hline & HighWire Press & http://highwire.stanford.edu/ \\
\hline & Latin America and the Caribbean (LILACS) & http://lilacs.bvsalud.org/en/ \\
\hline & $\begin{array}{l}\text { Medical Literature Analysis and Retrieval System Online } \\
\text { (MEDLINE) }\end{array}$ & http://www.nlm.nih.gov/pubs/factsheets/medline.html \\
\hline & Pubget & http://pubget.com/site/favicon.ico \\
\hline & PubMed Central (PMC) & http://www.pubmedcentral.nih.gov/ \\
\hline & Science Direct & http://www.sciencedirect.com \\
\hline & Web of Knowledge & http://wokinfo.com/ \\
\hline \multirow[t]{9}{*}{ Specialist databases } & Agricultural Biotechnology Network in Africa (ABNETA) & http://abneta.org/ \\
\hline & African Centre for Biosafety (ACBIO) & http://www.acbio.org.za/ \\
\hline & AgBioForum & http://www.agbioforum.org/ \\
\hline & Bi[bli]osafety database & http://bibliosafety.icgeb.org/search?SearchableText= \\
\hline & $\begin{array}{l}\text { Database of the Safety and Benefits of Biotechnology- } \\
\text { CropLife International }\end{array}$ & http://biotechbenefits.croplife.org/ \\
\hline & Greenpeace publications & http://www.greenpeace.org/international/en/publications/ \\
\hline & International Food Policy Research Institute (IFPRI) & http://www.ifpri.org/ \\
\hline & $\begin{array}{l}\text { International Service for the Acquisition of Agri-biotech Appli- } \\
\text { cations (ISAAA) }\end{array}$ & http://www.isaaa.org/ \\
\hline & Monsanto publications & $\begin{array}{l}\text { http://www.monsanto.com/products/Pages/biotech-technical- } \\
\text { publications.aspx }\end{array}$ \\
\hline Search engine & Google Scholar & http://scholar.google.com \\
\hline Other & $\begin{array}{l}\text { ProQuest (Dissertations \& Theses, Dissertations \& Theses } \\
\text { (PQDT) A\&l, Sociology, Sociological Abstracts) }\end{array}$ & http://www.proquest.co.uk/en-UK \\
\hline
\end{tabular}

database-specific, the short search strings were not specified a priori.

In cases where web searches and databases displayed large numbers of results in the order of relevance (e.g. Google Scholar), only the first 100 were evaluated. For databases that returned results of equal relevance (e.g. Web of Knowledge) and retrieved a high number of hits (in the order of thousands), a title screening was performed prior to an abstract screening.

\section{Article screening}

\section{Study inclusion criteria}

The following criteria were applied to each article to determine its possible suitability for further inclusion.

Language English (the language in which the reviewers were most proficient and also the language in which the majority of articles retrieved during the scoping exercise were written).

Timeline Published after 1994-the year when the first GM crop was commercially cultivated (on-going research was not included).

Population Farmers (understood to be farm owners, farm labourers and agricultural contractors) in developing and developed countries.
Intervention GM cultivation: any type of GM trait and any type of host crop.

Comparator Non-GM crop cultivation (both conventional and organic).

Outcomes Intermediary outcomes (amount of pesticide usage and income derived from GM crop cultivation) and final outcomes (number of pesticide-related poisonings and health-related expenditures).

\section{Screening process}

The primary hits retrieved were screened by each reviewer at the level of title and abstract, according to the Inclusion criteria. Each reviewer made a note of the exact search string(s) used for each database, along with the number of hits retrieved and the list of selected potentially relevant articles. The information was collected by a search coordinator. Those articles that the reviewers remained uncertain of their relevance to the Inclusion criteria were retained and included in the next step of the review process. To reduce reviewer bias, all articles deemed relevant by an individual reviewer were included in the subsequent full-text analysis.

Following the search and screening of the databases, a list was made (Additional file 1: Table S1) of articles that 
had been found to correspond to the Inclusion criteria. Next, the full text of every article in this list was sought, either directly from the database in which they were identified or provided by the authors upon request. Specific articles were moved to a separated list (Additional file 2: Table S2) if the full text was not immediately freelyavailable, even after attempts to contact the corresponding author. In some cases, the full texts became available at a later date in the review process. These full texts were evaluated for suitability but none was found to be relevant. Such articles were therefore not included in the list of full articles to be analysed, nor did they take part in the Kappa Cohen test calculations.

To verify the consistency of selection between reviewers at the level of title and abstract screening a Cohen Kappa test is usually recommended and the protocol of this systematic review included one at this step. The threshold previously set was 0.6. However, a classical Cohen Kappa test could not be performed at this step due to the combination of the search and screening done independently by each reviewer. However, to avoid losing potentially relevant articles, all articles selected during individual screening were included in the full text analysis step.

To test the reviewers' screening consistency at the level of title and abstract, a Cohen Kappa test was undertaken as a separate exercise: all three reviewers ran a full-string search within Science Direct and exported the first 100 hits that were then analysed using the inclusion criteria. The 100 hits were divided between the reviewers to evaluate for potential inclusion: reviewer 1-all hits; reviewer 2-the first 50 hits; reviewer 3-the second 50 hits. A Cohen Kappa test was then undertaken to determine the degree of agreement between reviewers 1 and 2, and between reviewers 1 and 3, respectively. The results indicated weak agreements between reviewers 1 and $2(0$. 498) and reviewers 1 and 3 (0.36). As both results were lower than the 0.6 threshold established in the protocol, a discussion ensued between all reviewers to clarify how to consistently apply the inclusion criteria. The reason for the initial poor results of the Cohen Kappa was determined as being the tendency of one reviewer to include articles when the title and abstract could not supply sufficient information, whilst the other two reviewers tended to reject them. As this did not affect the inclusion criteria, they did not require a re-evaluation. However, all reviewers then agreed that it was necessary to retain those articles for which the title and abstract did not supply sufficient information to confidently assess their relevance to the inclusion criteria.

All full-text articles were divided amongst the reviewers for a full-text analysis using the inclusion criteria, with each article evaluated by two reviewers. At the end of the full-text analysis, the Cohen Kappa test was repeated; the results $(0.615$ and 0.612$)$ demonstrated an acceptable degree of agreement between the reviewers.

\section{Potential effect modifiers and reasons for heterogeneity}

Potential effect modifiers and reasons for heterogeneity were established following discussions amongst the reviewers. Data was then extracted for each potential effect modifier. The modifiers were categorised into larger groups, each with several subgroups, as follows.

1. Type of intervention (GM crops):

1.1. Number of crops analysed

1.2. Type of crop

1.3. Type of trait introduced

1.4. Type of pesticide(s) applied

2. Context:

2.1. Country

2.2. Location

2.3. Agro-ecological climate (average temperatures, precipitation, etc.)

2.4. Weather (during the study cultivation)

2.5. Pest pressure

2.6. Irrigated/rain-fed

3. Farm and farmer profiles:

3.1. Age

3.2. Gender

3.3. Education

3.4. Farm size

3.5. Areas cultivated with GM/non-GM crops

3.6. Use of pesticide protective measures (if relevant)

4. Institutional context:

4.1. Agricultural subsidies

4.2. Agricultural credits

4.3. Price control policies

4.4. Agricultural extension services

5. Study design:

5.1. Methodology employed

5.2. Frequency and period of data collection

5.3. Sample size

\section{Data extraction}

Data extraction from all studies was done by one reviewer, and then subsequently reviewed and validated by a second reviewer (see Additional file 3: Appendix 1). For each study, the variables included the main findings for the intermediary and final variables, as well as the potential effect modifiers and reasons for heterogeneity listed above. 


\section{Data quality assessment}

The quality of the final studies was evaluated using a set of 8 questions requiring dichotomous answers (Yes/No). In some cases the question was not relevant to a particular study, in which case N/A was given as the response to the question. The overall purpose of the quality assessment was to assess causality, both for the intermediary and final variables (Table 2). Amongst the questions used, one in particular needed further explanation: "Did the study test differences between GM adopters and non-GM adopters?" does not refer to the presence of the comparator group, but to the testing for the effects of self-selection bias (when a technology is being adopted freely and not assigned randomly in the course of an experiment). In the latter case, self-selection introduces bias through the presence of further variables that could have an impact on the final results aside from the variables studied (for example, the income obtained from the cultivation of GM crops may not be primarily the result of the technology but also the result of the entrepreneurial drive of the adopters).

\section{Results}

\section{Included studies}

The analysis of 20 databases and 10 reviews returned 4,870 hits, of which 200 articles were selected as relevant by title and abstract (Fig. 1). Of these 200, the full text of 180 articles was successfully retrieved and evaluated. Following full-text analysis, only 19 articles were identified as relevant. Some of these 19 articles showed sufficient similarities (year of the survey, location, authors, unit of analysis) to conclude that they derived from the same original study. In such cases (e.g. for a multi-year survey that resulted in several published articles), the article with the most comprehensive set of results was identified as the main article for data extraction, and the others retained as a source for supplementary information (Additional file 4: Table S3). Therefore, of the 19 articles selected for data extraction, only 9 described distinct studies (codified as studies \#1-\#9), of which 7 were relevant to the first research question, whilst 2 were relevant to the second (see Additional file 4: Table S3, Additional file 5: Table S4; Tables 2, 3, 4 for further details on the studies). In addition, 20 articles (10\% of the articles considered relevant by title and abstract) could not be immediately retrieved free of cost. Subsequently, of these 20, the full text of 4 articles was obtained, analysed by reviewers, and found not relevant for the systematic review (Additional file 2: Table S2).

\section{Characteristics of studies included}

The remaining nine studies were analysed to extract data according to the protocol [18]. The studies took place in a total of four different countries: China, India, Pakistan and South Africa. An overwhelming majority (8) of the studies concerned cotton, whilst one study focused on pre-production trials for rice. There were no studies focusing on more than one crop. In terms of cultivation study duration, the surveys and written data covered: 1 cropping season (3 studies), 2 seasons (3 studies), 3 seasons ( 2 studies) and even 4 seasons (1 study) (see Tables 3, 4). All the GM events were insect-resistant. Information concerning the types of pesticides used by farmers was generally absent with a few exceptions. There was one study that investigated pesticides in terms of the hazard categories to which they belonged according to World Health Organization definitions, and ranked and reported the amount of pesticides used in each category (study \#3, see Appendix 1). In addition, two other studies reported the most popular pesticide for the region surveyed [Emamectin (study \#4) and Cypermethrin (study \#7)] yet did not register the exact amounts used by farmers.

The grey literature was a source of a diverse range of publications included in data extraction: 13 peerreviewed articles, 2 working papers, 2 conference articles, 1 monograph and 1 doctoral thesis.

Regarding the funding sources, among the nine studies, with the exception of two studies that did not mention funding agencies or donors, funding for research generally came from academic and research institutions. Industry contributed to the funding in only one case (study \#2).

As mentioned previously, context is very important in measuring the impact of GM crop cultivation, as it can introduce confounding variables that can considerably change the conclusion drawn and the potential for generalisation of the results. In this systematic review, the main constituents of agricultural context were the weather (including climate and pest pressure), farm and farmers' characteristics, and institutional characteristics (agricultural credits and subsidies, price control policies, agricultural extension services, etc.). The study farms were subject to climate typical for the cultivation of cotton or rice. However, for studies that spanned more than 1 year, variations in weather conditions, especially rain, impacted the amount of pesticides applied and cultivation costs (especially due to the need for increased pesticide use and irrigation). Cotton bollworm infestation, for example, usually increases in a wet season, whilst a drought makes the use of irrigation necessary. Information about the weather helps to demonstrate causality between the cultivation of the GM crops and the increase/decrease of pesticides or increase/decrease of farm incomes. It also gives numerical results (statistics) a better understanding. If GM crop adopters receive 


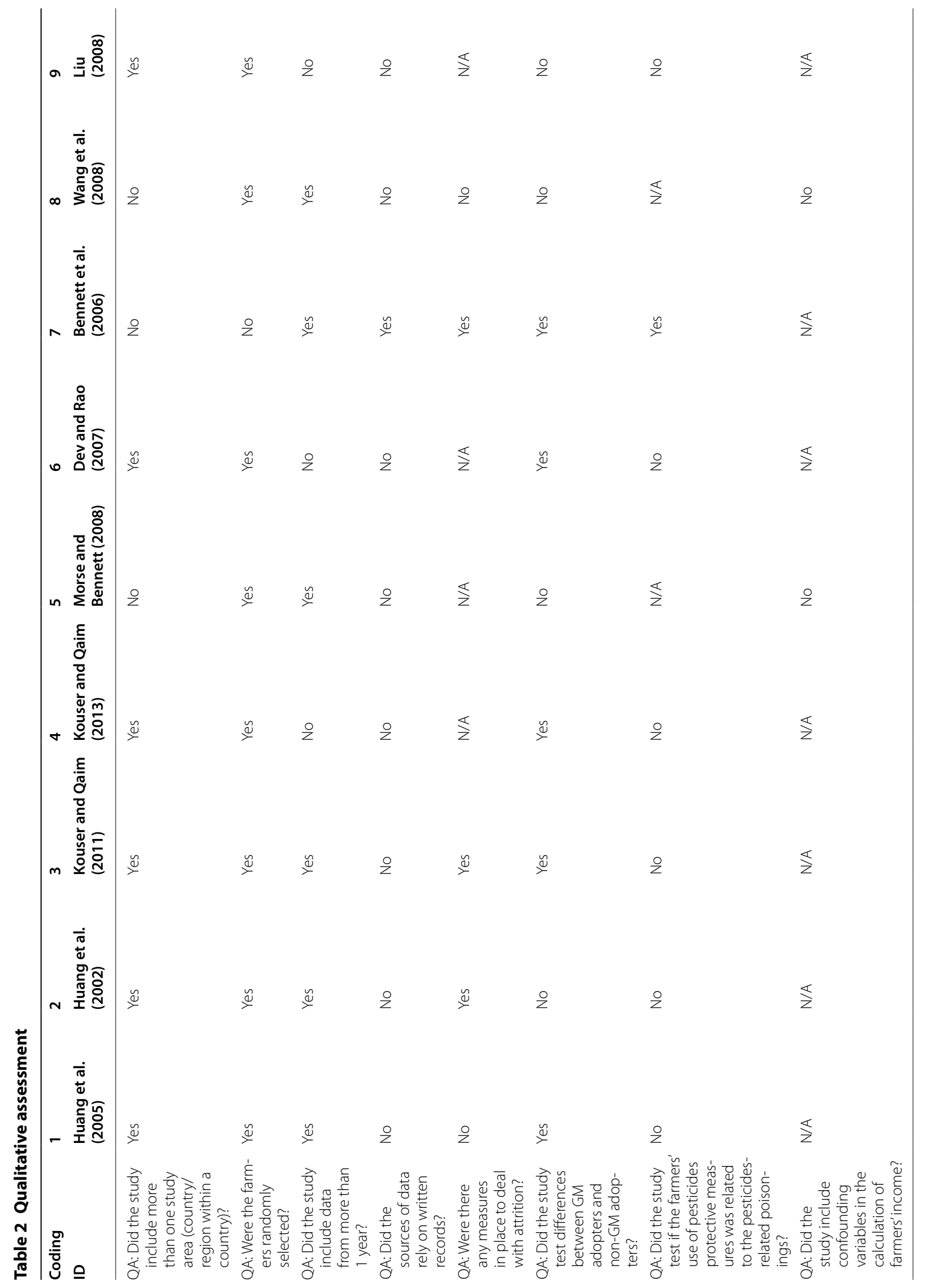




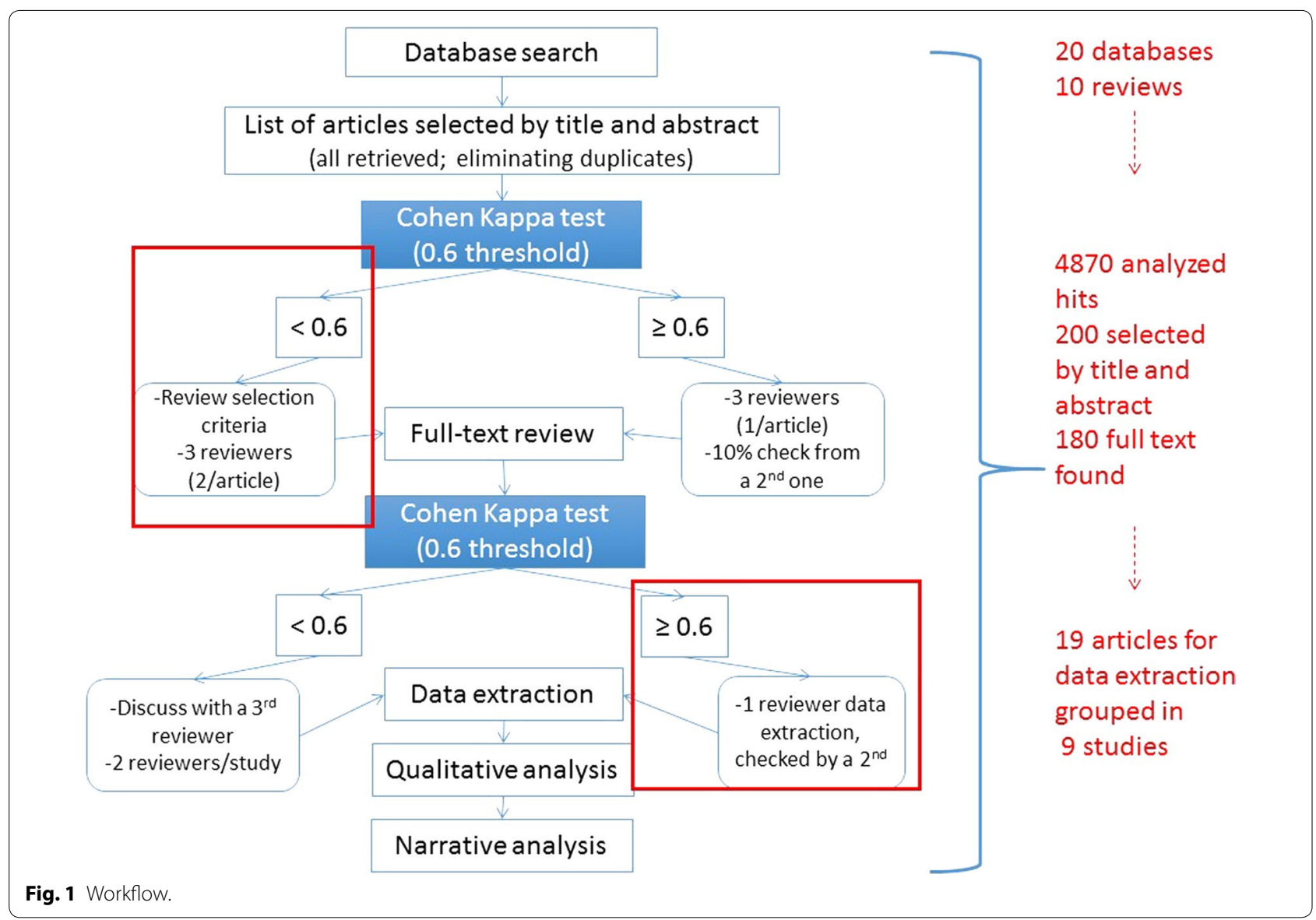

greater benefits than non-adopters, even in seasons with bad weather and increased pest pressure, it is a strong indication of the added value of the GM crops.

For the first research question, pest pressure was the primary concern, because the amount of applied pesticide is usually adapted by farmers according to the level of infestation observed. Two studies formally included pest pressure in the analysis (studies \#1 and \#9), three mentioned it only as a potential factor impacting the use of pesticides, whilst two studies did not mention pest pressure at all. Of the last two, study \#3 stated that no major climatic changes occurred for the period surveyed, and study \#6 stated that rainfall was $25 \%$ lower than usual during the research period. Therefore, no weather-related bollworm infestation was reported in the areas analysed. For the second research question, one study reported a drought for the period surveyed with no irrigation measures in place (study \#5), whilst the other reported neither weather nor irrigation information (study \#8).

In attempts to determine causality, the characteristics of farms and farmers are routinely registered in surveys of pesticide use or economic impact. In this case, the question could be reformulated as: "is the use of pesticides or household income determined by the new technology alone or by the characteristics of the users?" Amongst the farmer characteristics most registered in surveys, the current review focused on age, gender and education, whilst those of the farm included size, area cultivated with GM/non-GM crops, and the use of any pesticide protective measures when spraying. Although age, gender and education are amongst the variables that are commonly registered in surveys, only a few studies investigated their connection with the technology (studies \#2, \#3 and \#7). The same studies were also the only ones that analysed whether the benefits of GM crop adoption depended upon farm size. The area cultivated with GM or non-GM crops was generally registered with two exceptions (studies \#4 and \#9). With the exception of study \#5 that used a before-after type of impact study (i.e. non-GM crop cultivation followed by GM crop cultivation on the same area), the other studies surveyed farmers cultivating GM and non-GM crop concurrently. Only study \#7 tested if farmers used protective measures when spraying pesticides.

As positive results obtained with an agricultural technology such as GM crops can be attributed to price 


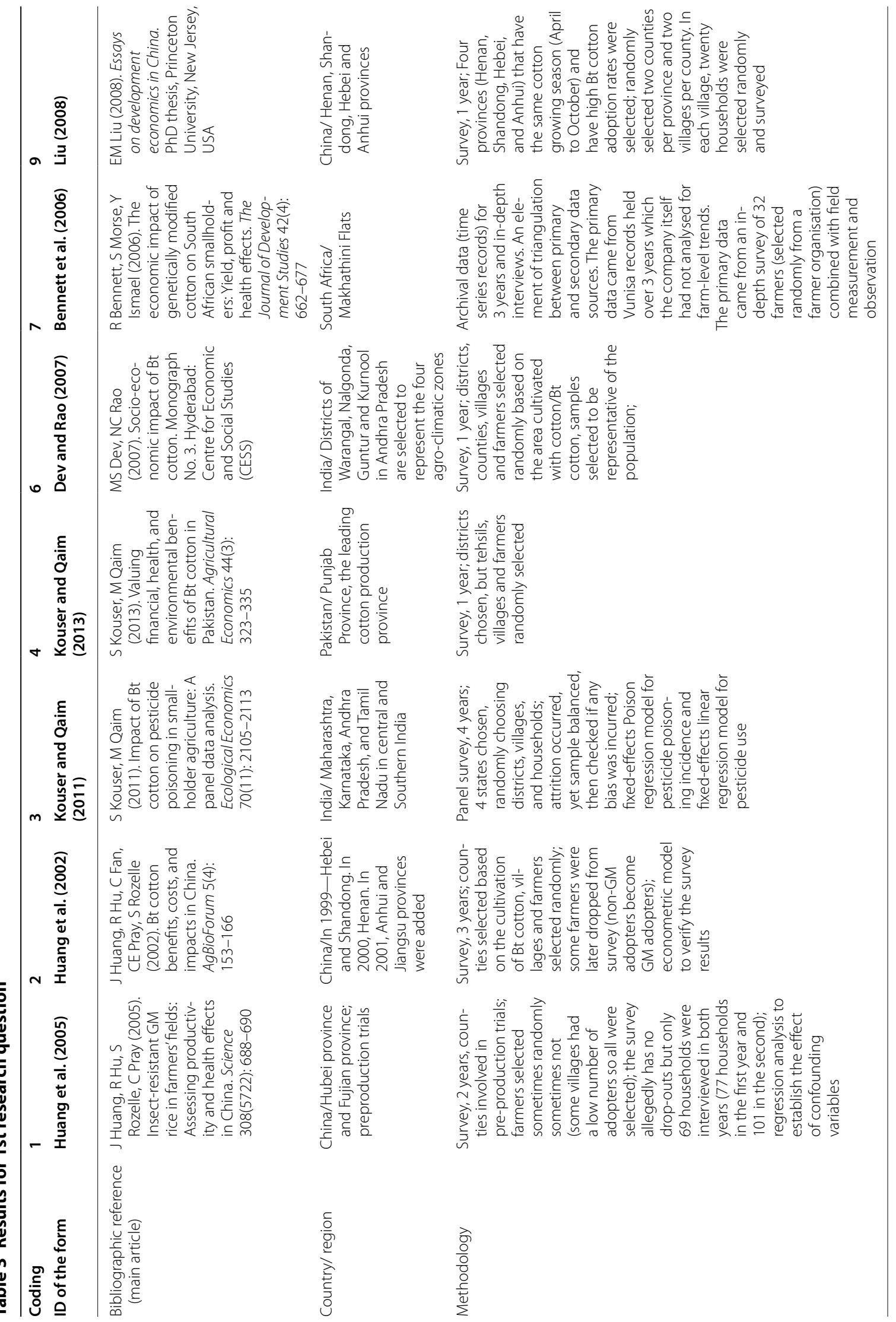




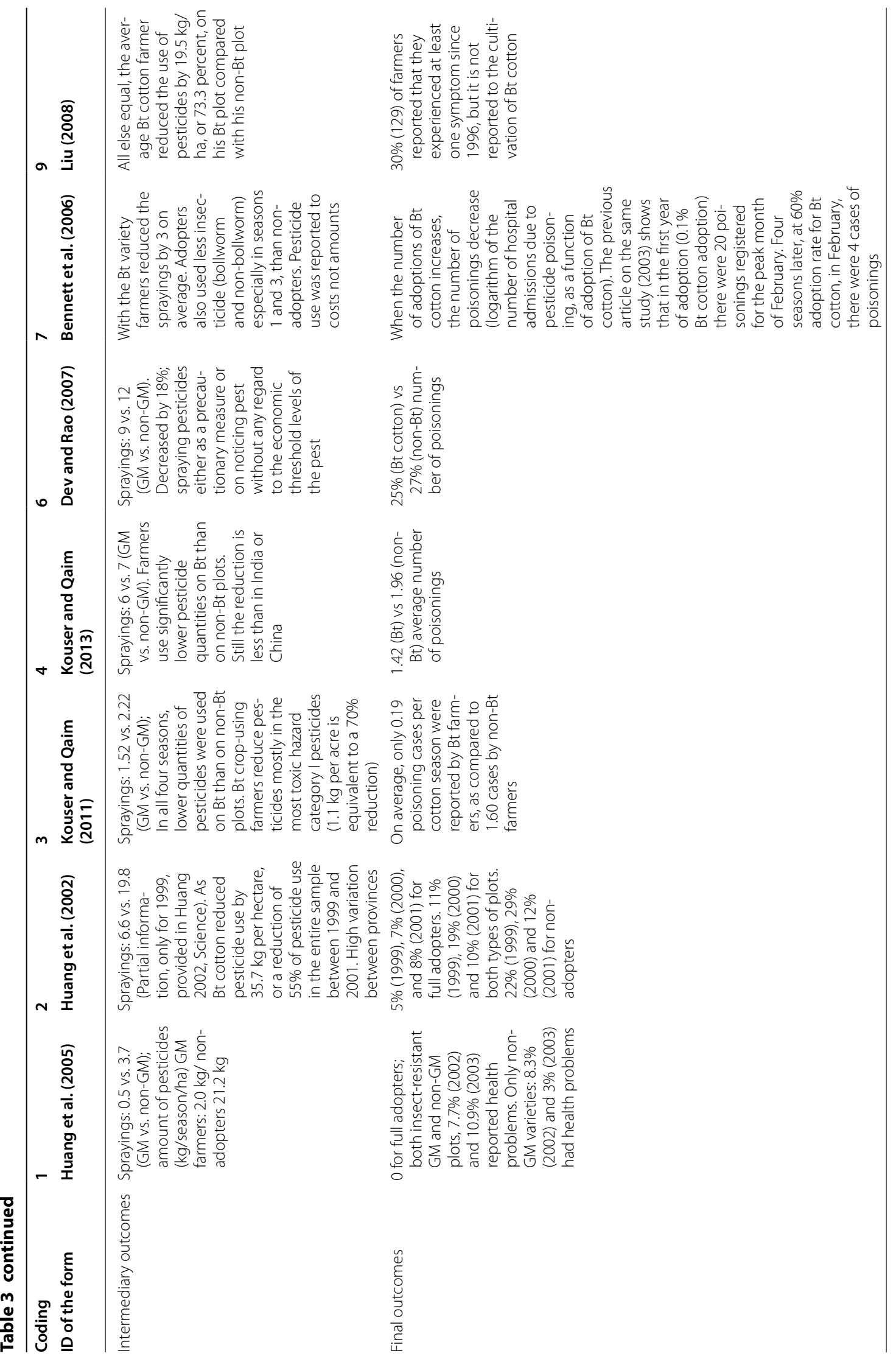




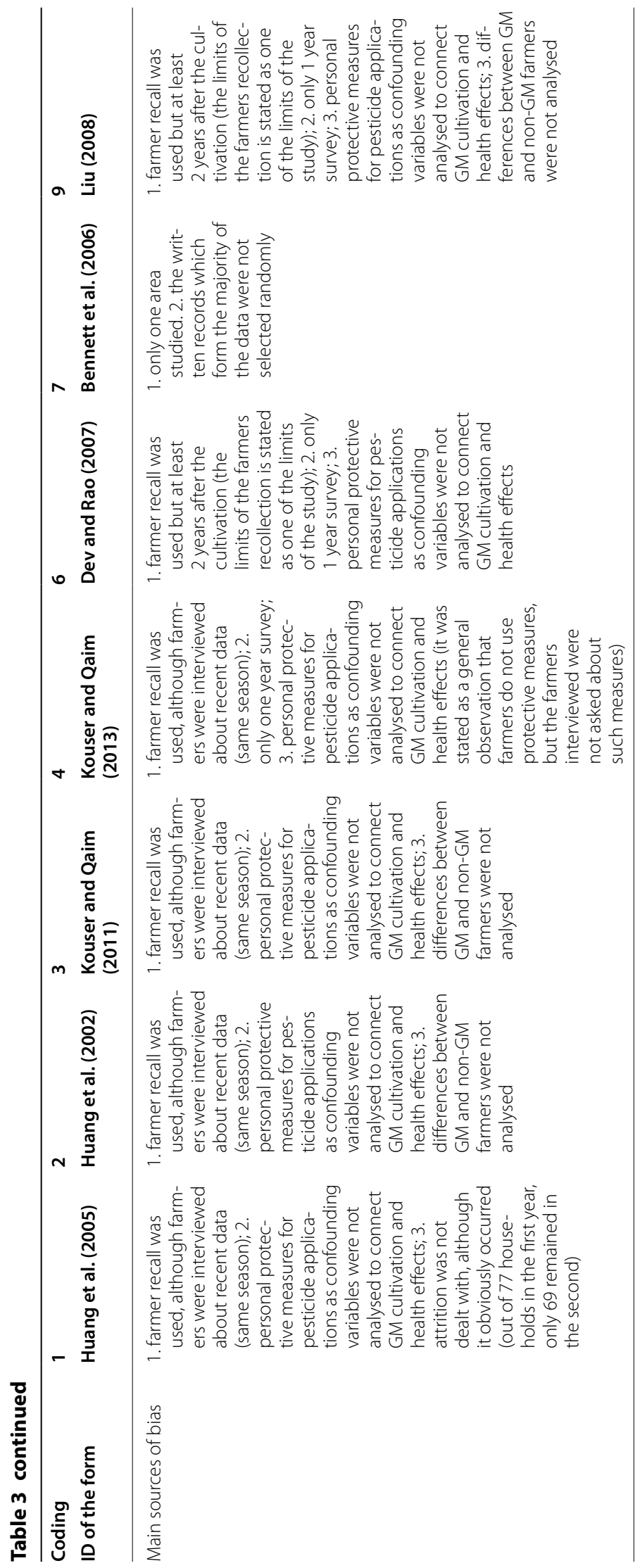




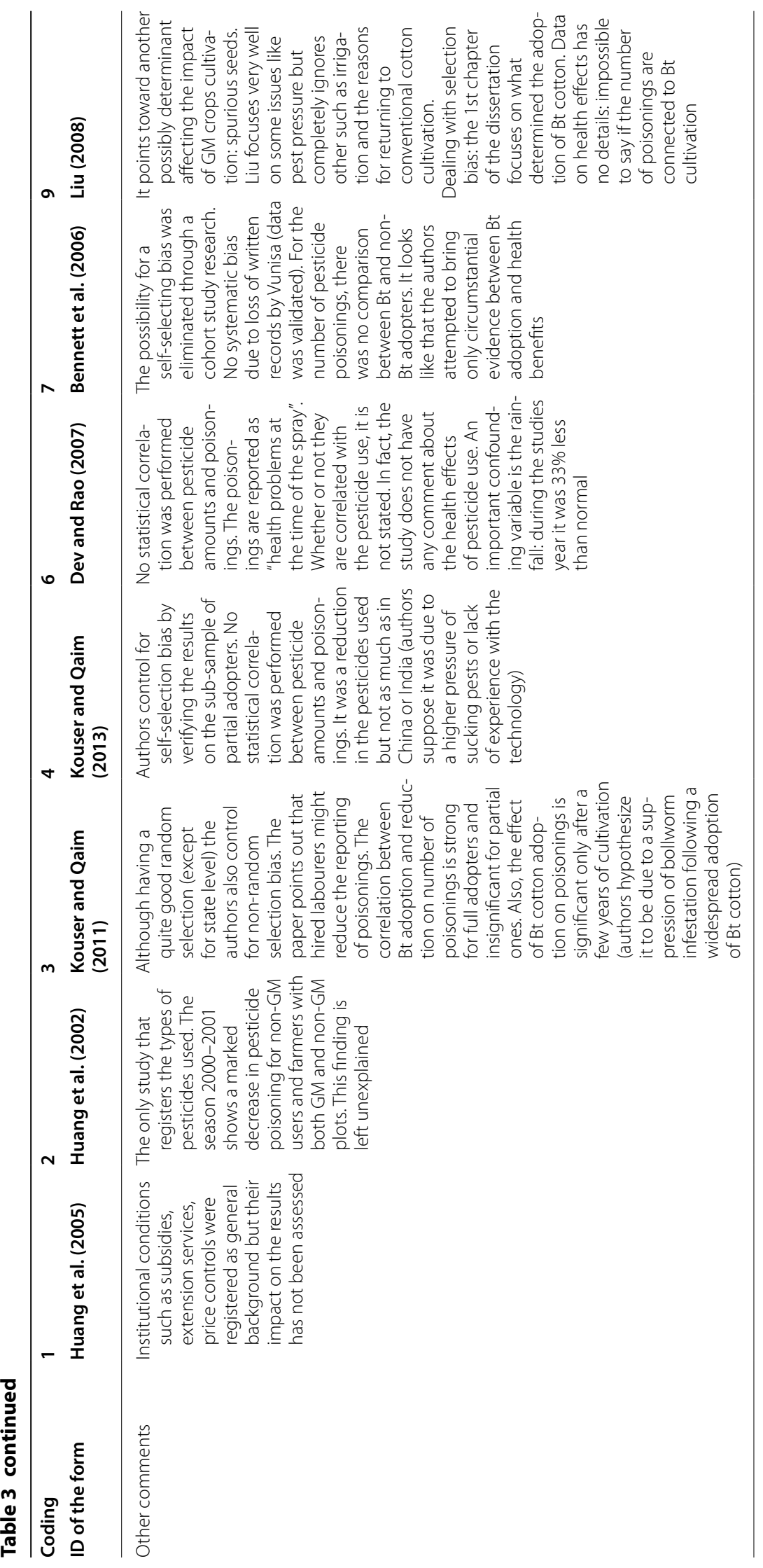




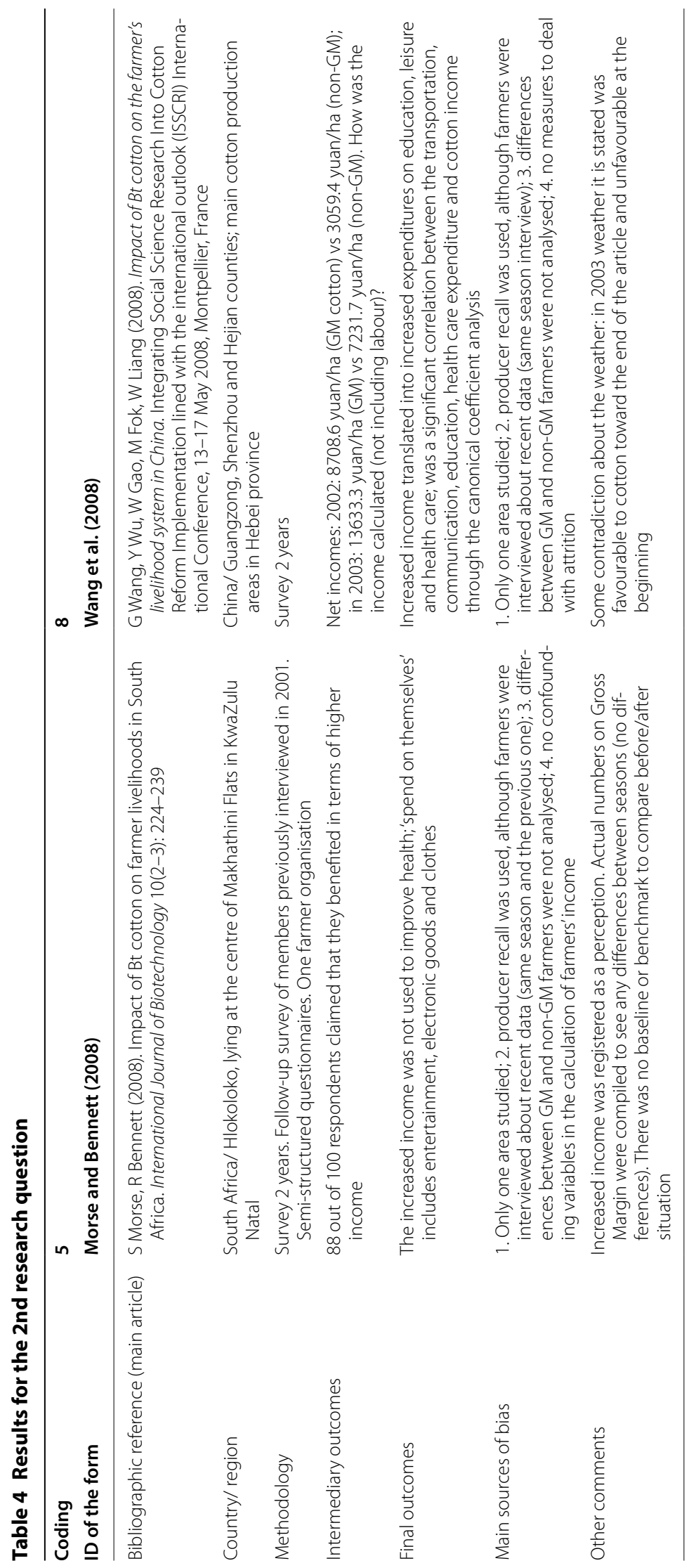


controls for seeds, credits or subsidies for their cultivation, as well as to the training of farmers in the proper use of the technology, data was also extracted concerning these institutional characteristics. Of the nine studies, six did not mention agricultural subsidies, seven did not mention agricultural credits, and eight did not mention if price control policies were in place. The role of extension services was reported in only two of the studies.

\section{Systematic review limitations}

The literature search only focused on English language articles and freely-available sources. As the results of the pre-protocol screening pilot [18] indicated, the number of articles published in Spanish or Portuguese was much lower than those in English. In addition, studies concerning GMO impacts tend to be published in English to garner the greatest readership. Therefore, it was considered that the limitation posed by the exclusion of studies in Spanish or Portuguese (even if the cultivation of GM crops is extensive in areas where such languages are spoken) was minimal. A possible concern, however, was the inability to conduct searches for relevant articles published in Standard Chinese/Mandarin and the present research has no way of evaluating this limitation. Furthermore, due to the search being limited to only freelyavailable sources, 16 articles were not retrieved, even after contacting the authors. As some of these did not have comprehensive abstracts, it was difficult to assess if the articles were likely to contain relevant data.

Furthermore it was not clear to what extent poor reporting of the results and the methodology used in the retrieved studies may have introduced bias into the analysis of those studies. However, for studies that spanned more than 1 year and for which more than one publication was produced, it was possible to identify a substantial amount of supplementary information to help reduce bias from poor reporting. In addition, a further limitation was the fact that ongoing research was deliberately not included in the protocol for this Systematic Review [18].

\section{Findings}

In relation to the first research question, all of the nine studies reported a decrease in the amount and number of pesticide sprayings during the cultivation of GM crops (Table 3). For the final variable, the number of poisonings, all studies reported a reduction in the number of pesticide-associated poisonings during the cultivation of the studied GM crops. However, the results varied considerably for both pesticide use and number of poisonings. Concerning pesticide quantities, the studies directly reported reductions ranging from $18 \%$ in India (study \#6) to over $70 \%$ in China (study \#9), and reductions in the number of sprayings by almost 13 in China (study \#2) to only one in India (study \#6). Sometimes there were significant variations in pesticide use between different areas included in a study (study \#2). For the number of poisonings, the cases varied from 0 for farmers that had only GM crop plots (in China; study \#1) to $25 \%$ of GM crop-cultivating farmers (in India; study \#6). As reporting was not uniform between studies, no easily comprehensible graphs could be prepared to demonstrate the variation in results. Most studies provided information on both the amount of pesticide used and the number of sprayings, however only a few studies provided one variable for measuring pesticide use. For one study (study \#9), the number of pesticide poisonings was not even separated between the GM and non-GM crop adopters, nor was it reported per year; it was simply reported as a percentage of farmers who, for the period analysed, reported pesticide-related health problems.

The variation in the amounts of pesticides used (within the same study or between studies) has been attributed by the study authors to: (1) climatic conditions driving fluctuations in the prevailing pest populations; (2) the use of lower quality seeds, either through the purchase of spurious seeds or through the practice of saving seeds; (3) the development of resistance in the target pests (study \#2); (4) the rise of secondary pests; (5) differences in extension services (study \#4), or; (6) different farmer attitudes to risk (risk-averse farmers using more pesticides; study \#9). However, possible confounding variables in the use of pesticides were not explored in the majority of the studies analysed. Only two studies established a strong correlation through regression analysis between GM crop cultivation and a reduction in pesticide use (studies \#1 and \#3). Further correlations between the use of pesticides and pesticide-associated poisonings were pursued in only two studies (studies \#2 and \#3).

For sources of bias, a qualitative analysis (Table 2) revealed that, with one exception (study \#7), the studies based their analysis on farmer recall. An attempt was made in four studies (Table 3) to minimise bias by conducting each survey at the end of the agricultural season. A major bias, however, was the fact that personal protective measures for pesticide applications as confounding variables were not analysed as an element connecting GM cultivation and health effects (with one exception, study $\# 7$ ). In only two cases (studies \#2 and \#4), it was generally stated that farmers in the study region did not usually use protective measures when spraying, but relevant questions were not included in the surveys to verify this fact. Further, three studies did not test these differences between GM and non-GM crop adopters, whilst another three reported the results of only one cultivation year.

There were studies that introduced new perspectives to consider. For example, study \#2 was the only study that 
analysed the types of pesticides used and how the cultivation of GM crops impacted each category of pesticides. GM crops appeared to have the strongest impact (reduce the amount used) on the most toxic category of pesticides (e.g. organophosphates, pyrethroids). Given that all of the final studies reported a reduction in pesticide use with the cultivation of GM crops, it would be worth further exploring the impact on different categories of pesticides, to definitively establish whether or not GM crop cultivation results in a reduction in the use of highly toxic pesticides. In addition, as agricultural impact studies generally have to work with available acreages of the technology instead of the preferred random assignment, and that self-selection bias (when individuals become members of a specific study group due to autonomous farm-based decisions, e.g. by choosing to cultivate GM crops) was not included as a major source of bias to track, two of the studies conducted analyses to control for non-random selection bias (studies \#3 and \#7). Furthermore, study \#9 used regression analysis to evaluate the behavioural characteristics of farmers, namely attitudes towards risk, as another potential main determinant in the use of pesticides. Farmers who were more risk averse continued to use high amounts of pesticides even after adopting GM crops. Training can correct the tendency, but needs to be repeated at regular intervals to avoid being lost over time. In addition, the study found that one of the reasons for the risk averse behaviour was the unreliable quality of the seeds. Either the presence of spurious seeds or variations in the quality of the seed of approved commercial events left farmers distrustful in the seeds that they purchased, thereby resulting in higher amounts of applied pesticides as a guarantee of harvest quality. Spurious seeds, as a possible source of quality reduction of the GM seeds and thus requiring greater numbers of pesticide spraying, were only reported in studies \#2 and \#4. Another confounding variable reported by authors concerned the possibility that the use of hired labourers may have affected the number of poisonings, however this hypothesis was not further explored (study \#3).

For the second research question, only two studies were found to be relevant (Table 4). Both studies determined that the cultivation of GM crops increased farmers income without, however, separating the reporting between farm owners and labourers. Regarding the uses for the extra income, study \#5 found that farmers mostly invest in their children's education, in cotton cultivation (unspecified) or to repay debt, respectively. Health was not reported as an expenditure category, even as part of the lower-rated categories such as "spend on themselves". In study \#8, a canonical coefficient analysis found that education was again a priority in household expenditure for the extra income accrued, along with leisure and health care.

Both of these studies (study \#5 and study \#8) had several sources of bias. They focused on only one cultivation area, although this is not unusual for in-depth studies. Both studies did not test differences between GM and non-GM crop adopters (study \#5 used a before-after methodology). Both also relied on farmer recall for relevant data. Moreover, study \#5 undertook only one round of interviews regarding the previous two agricultural seasons. If farmers are interviewed immediately at the end of the agricultural season with questions pertaining directly to that particular season, the information gathered is still fresh in their minds and is therefore more accurate, thereby reducing recall bias. In addition, study \#5 did not consider confounding variables in the calculation of farmers' income, while study \#8 had no measures to deal with survey sample attrition. Additional shortcomings included establishing increased income by reporting the perceptions of farmers instead of by direct measurement (study \#5), and contradictory statements over the weather during the GM crop cultivation (study \#8).

\section{Conclusion}

In general, the amount of pesticides applied in GM crop cultivation was lower than in non-GM crop cultivation, even though in the majority of cases the results were based on questionnaires (which may risk recall inaccuracies). The link between GM crop cultivation and a reduction in number of pesticide poisonings should be considered as still circumstantial, due to the absence of relevant confounding variables or statistical analyses to support the conclusions. The cultivation of GM crops also appeared to increase household income. It was however not possible to accurately determine what proportion of the extra income is devoted to dealing with health issues. Farmers rated the education of their children as the most important household expenditure overall.

Further studies could benefit from addressing the identified methodological shortcomings reported in this systematic review. It would for instance be useful to take cognisance of the following:

1. Using surveys as the sole basis of data-gathering should be done with caution. Written records are usually the most reliable source of data, but in the developing world these are often lacking. One way to increase the accuracy of survey data, in the absence of written records, would be to take records immediately following crop harvest. The data should also be submitted to a form of validation (for example, triangulation as was done in study \#7). 
2. Although open-ended questions can be used to gather valuable data, concrete questions can more accurately establish a deterministic relationship between variables. Statistical validation of data (correlations between independent and dependent variables, statistical significance for parameters, etc.) can provide a solid basis for further analyses.

3. Confounding variables have always been a delicate point in surveys, and research in general. If overlooked, the causal relationship may be questioned; if too many are considered, it may make the research unmanageable. Study analyses should focus on identifying appropriate confounding variables so that they can be eliminated or minimised. For example, in relation to the first research question of this review, one essential confounding variable seldom considered in the analysed studies was the use of protective wear for farmers when spraying pesticides.

The foregoing recommendations acknowledge however that studies relying on widespread GM crop cultivation for data acquisition may have difficulty in identifying ideal test/survey areas. Study design may have to be adapted accordingly, with a possible consequence of a reduction in the power of their analyses. Many of the studies that entered the present data extraction, for example, were of high quality, considering the limitations faced by agricultural surveys in general; the unwillingness of some farmers to participate in surveys; the lack of any written data, and; the lack of funding to undertake surveys with numerous variables, to name but a few.

In addition, the review found that information relevant to the non-food impacts of GM crop cultivation on farmers' health is generally available at no cost (either free to download or provided by authors when requested).

GM crops are a highly politicised issue and their potential impacts are presented as facts before being thoroughly studied. For example, there are reports that tend to take at face value the fact that a reduction in pesticides use associated with the cultivation of GM crops leads also to a reduction in occupational pesticides poisonings. This review indicated however, that there are several variables that could impact such possible cause-effect relationships and that further research needs to be undertaken to substantiate any such claims.

Overall, the present systematic review uncovered weak connections between GM crop cultivation and (1) pesticide poisonings, (2) income, and to any subsequent general health improvements. A possible reason for the lack of strong connections may be that they were not the main objectives of the studies analysed. In such a case, new studies aiming to expressly generate clearer evidence linking the intermediary and final variables studied in this systematic review would be very useful.

\section{Additional files}

Additional file 1: Table S1. Articles selected by title and abstract.

Additional file 2: Table S2. Articles not found full text.

Additional file 3: Appendix 1. Data extraction.

Additional file 4: Table S3. All articles published on the studies analysed.

Additional file 5: Table S4. Articles excluded.

\section{Abbreviations}

ICGEB: International Centre for Genetic Engineering and Biotechnology; GM: Genetically modified; GMO: Genetically modified organism; ISAAA: International Service for the Acquisition of Agri-biotech Applications.

\section{Authors' contributions}

All authors read and approved the final manuscript.

\section{Author details}

${ }^{1}$ Biosafety Unit, International Centre for Genetic Engineering and Biotechnology (ICGEB), Trieste Component, Padriciano, 99, 34149 Trieste, Italy. ${ }^{2}$ Biosafety Unit, ICGEB, Cape Town Component, UCT Campus, Anzio Rd Observatory, Cape Town 7925, South Africa. ${ }^{3}$ Present Address: STS-Institute of Science, Technology and Society Studies Alpen-Adria-Universität Klagenfurt, Vienna, Graz, Schlögelgasse 2, 8010 Graz, Austria.

\section{Acknowledgements}

The authors would like to thank two anonymous reviewers and the Editor-inChief, Andrew S. Pullin, for their helpful comments.

\section{Compliance with ethical guidelines}

\section{Competing interests}

The authors declare that they have no competing interests. The systematic review was funded by the ICGEB, both from its core funding, as well as from external funds raised by its Biosafety Unit.

Received: 16 October 2014 Accepted: 16 July 2015

Published online: 03 August 2015

\section{References}

1. James C (2014) Global status of commercialized Biotech/GM crops: 2014. ISAAA Brief No. 49. Executive Summary. ISAAA, Ithaca

2. Brookes $G$, Barfoot P (2013) The global income and production effects of genetically modified (GM) crops 1996-2011. GM Crops Food. 4:74-83

3. Kleter G, Bhula R, Bodnaruk K, Carazo E, Felsot ES, Harris CA et al (2007) Altered pesticide use on transgenic crops and the associated general impact from an environmental perspective. Pest Manag Sci 63:1107-1115

4. Brookes G, Barfoot P (2014) GM crops: global socio-economic and environmental impacts 1996-2012. PG Economics Ltd., Dorchester

5. Carpenter JE (2011) Impact of GM crops on biodiversity. GM Crops. 2:7-23

6. Fawcett R, Towery D (2003) Conservation tillage and plant biotechnology: how new technologies can improve the environment by reducing the need to plow. The Conservation Technology Information Center (CTIC), Indiana

7. Benbrook CM (2012) Impacts of genetically engineered crops on pesticide use in the U.S. - the first sixteen years. Environ Sci Eur 24:1-24

8. Gurian-Sherman D (2009) Failure to yield. Evaluating the performance of genetically engineered crops. Union of concerned scientists 
9. Séralini GE, Mesnage R, Clair E, Gress S, de Spiroux Vendômois J, Cellier D (2011) Genetically modified crops safety assessments: present limits and possible improvements. Environ Sci Eur 23:1-10

10. World Health Organization. Frequently asked questions on genetically modified foods. http://www.who.int/foodsafety/areas_work/food-technology/faq-genetically-modified-food/en/. Accessed 28 Mar 2015

11. Hossain F, Pray CE, Lu Y, Huang J, Fan C, Hu R (2004) Genetically modified cotton and farmers' health in China. Int J Occup Environ Health. 10:296-303

12. Hoddinott J (2012) Agriculture, health, and nutrition: toward conceptualizing the linkages. In: Shenggen F, Pandya-Lorch R (eds) Reshaping agriculture for nutrition and health. IFPRI 2020 Book. International Food Policy Research Institute, Washington, DC, pp 13-20

13. Thirtle C, Beyers L, Ismael Y, Piesse J (2003) Can GM-technologies help the poor? The impact of Bt cotton in Makhathini Flats, KwaZulu-Natal. World Dev. 31:717-732
14. European Food Safety Authority (2010) Application of systematic review methodology to food and feed safety assessments to support decision making. EFSA J 8:1637-1727

15. The Cochrane Collaboration (2011) Cochrane handbook for systematic reviews of interventions version 5.1.0. In: Higgins JPT, Green S (eds)

16. Petticrew $M$, Roberts $H$ (2006) Systematic reviews in the social sciences: a practical guide. Blackwell, Hoboken

17. Centre for Evidence-Based Conservation (2013) Guidelines for systematic review in environmental management. Version 4.2. Environ Evid

18. Racovita M, Obonyo DN, Craig W, Ripandelli D (2014) What are the nonfood impacts of GM crop cultivation on farmers' health? Environ Evid. 3:

\section{Submit your next manuscript to BioMed Central} and take full advantage of:

- Convenient online submission

- Thorough peer review

- No space constraints or color figure charges

- Immediate publication on acceptance

- Inclusion in PubMed, CAS, Scopus and Google Scholar

- Research which is freely available for redistribution

Submit your manuscript at

www.biomedcentral.com/submit

C Biomed Central 